\title{
Strain rate influence on mechanical behavior of a single wire entangled material
}

\author{
Sandra Guérard $^{1, *}$, Jérémie Girardot ${ }^{1}$, Philippe $\operatorname{Viot}^{1}$ \\ ${ }^{1}$ Arts et Métiers ParisTech, Univ. Bordeaux, Bordeaux INP, I2M, UMR 5295, F-33000 Bordeaux, France
}

\begin{abstract}
In a global context of energy saving, the ratio stiffness - mass is a key parameter for design of mechanical structures. To deal with this major concern, sandwich materials are finding an increasing use: the skins are designed to resist tensile and compressive stresses while the core needs to gather lightweight, shear stresses resistance and high mechanical energy absorption capacities. Firstly made of balsa wood, the core is nowadays classically realized using architectured materials (cellular materials, honeycombs, entangled materials, etc.). Entangled materials are architectured materials with tuneable properties, depending of the dedicated application. Several entangled materials already exist such as mineral or metallic wool; some of them are made of a single ductile metallic wire, entangled in all directions so that the final material becomes a porous continuous media. Such materials, which combine lightness and ductile behaviour, seem to be perfect candidates to dissipate energy during an impact. Compared to conventional materials such as balsa wood or honeycomb, a large amount of energy is indeed dissipated by friction coming from the numerous contacts due to the entanglement. The global aim of this work is focused on the study of energy dissipation mechanisms involved during impact as well as the correlation between architectural parameters of the material (wire diameter and material, volume fraction, etc.) and macroscopic behaviour. The first step that is presented here consists of an experimental investigation using dynamic compression tests to study macroscopic parameters (wire diameter, volume fraction, etc.) on absorbed energy.
\end{abstract}

\section{Introduction}

In the way of looking for new material alternatives for engineering applications, the single wire entangled material (SWEM) (see Fig 1), also socalled "metal rubber" [1] has been widely studied during the last decades [2]. Applications can be various and be found in vibration absorbers, biocompatible parts or fluid filtering. Its porous geometry and specific intern architecture produce interesting specific mechanisms under compression loading.

Static compression [6-8], hysteresis [3], Poisson's function [4] and damping capacity [5] are mechanisms analysed regarding the manufacturing process of the SWEM which used a multiple coil geometry and confined compression to obtain a cylindrical sample [6,9]. Wires are mostly metallic with steel, aluminium or nickel-titanium alloys. In [4], a polyamide was used to obtain reversible hysteresis thanks to the low stiffness of the wire.

Although the material behaviour is already analysed under static and dynamic loadings, the dynamic part is only dealing with vibrations. In general, results show that this material has a strong interest in terms of damping characteristics. No impact or high speed compression has already been performed on this kind of material.

Concerning the shock absorption application, foam-like material such as metallic or polymer foams, syntactic foams, ceramics, aerogels or honeycomb structures are mainly based on one irreversible mechanism: the hyperelasticity inducing high strains [10]. This irreversible deformation comes from buckling mechanisms at the microstructure level. The present SWEM material has also an architecture where plastic strains occur especially due to its metallic ductile wire. An additional dissipative friction mechanisms coming from the numerous contacts inside the material are also a source of absorption. The main purpose of the subject is to evaluate the part of friction in shock absorption of SWEM material.

In this work, it is proposed to observe the compressive mechanical response of a single entangled wire sample with two different densities. A ductile copper wire is used. A Hopkinson bar device is used to perform a high speed loading and results are comparing to static compression to evaluate strain rate effects. As it is a preliminary work, a discussion gives some proposals for further investigation.

Corresponding author: $\underline{\text { sandra.guerard } @ \text { ensam.eu }}$ 


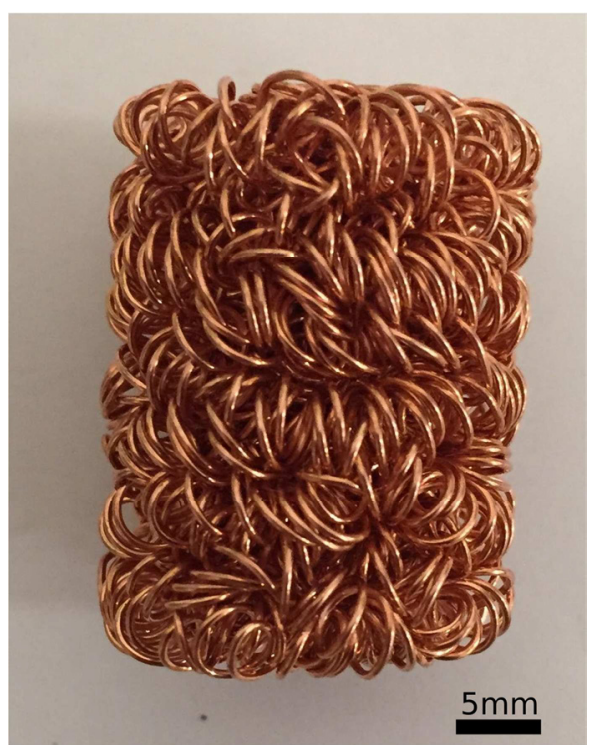

Fig. 1. Picture of single copper wire entangled sample.

\section{Material \& Methods}

\subsection{Characteristics of the specimens}

A copper bare wire with a diameter of $500 \mu \mathrm{m}$ was used to prepare the entangled wire preforms. Firstly, volume fraction and dimension of specimens have been chosen. In order to evaluate the influence of volume fraction on the dynamical response of entangled structures, 2 volume fractions were chosen: $\mathrm{Vf}=17 \%$ and $\mathrm{Vf}=25 \%$. Cylindrical shape specimens were chosen to facilitate manufacturing process. Outer diameter was fixed to $24 \mathrm{~mm}$ and height to either $31.50 \mathrm{~mm}$ for quasi-static (QS) tests or $7.00 \mathrm{~mm}$ for dynamic (SHPB) tests. All characteristics of specimen are summarized in Table 1.

Table 1. Characteristics of specimens (dimensions are given as mean value \pm 2 std)

\begin{tabular}{|l|c|c|c|c|}
\hline & \multicolumn{2}{|c|}{ QS tests } & \multicolumn{2}{c|}{ SHPB tests } \\
\hline Volume Fraction & $17 \%$ & $25 \%$ & $17 \%$ & $25 \%$ \\
\hline Diameter (mm) & 24.3 & 24.3 & $24.3 \pm$ & 24.3 \\
& \pm 0.3 & \pm 0.4 & 0.3 & \pm 0.5 \\
\hline Height (mm) & $\begin{array}{c}31.5 \\
\pm 0.6\end{array}$ & $\begin{array}{c} \pm 0.9 \\
\pm 0.3\end{array}$ & $\begin{array}{c}7.7 \pm \\
0.2\end{array}$ & $\begin{array}{c}7.7 \pm \\
0.02\end{array}$ \\
\hline $\begin{array}{l}\text { Initial wire length } \\
(\mathrm{mm})\end{array}$ & 12350 & 18155 & 2750 & 4040 \\
\hline
\end{tabular}

Finally, to assess repeatability of the results, 6 specimens for each configuration were prepared.

\subsection{Manufacturing process}

Manufacturing of the samples was made following Tan et al. [6] protocol, as presented in Fig. 2 and can be summarized as follows. The copper wire, which length was firstly calculated from chosen volume fraction and diameter and height (Fig 2a), has been initially encircled into a $3 \mathrm{~mm}$-diameter rod by distorting and twisting the wire (Fig 2b). The springlike segment has then been stretched so that the distance between two adjacent spirals is equal to the value of the diameter of the rod (here $3 \mathrm{~mm}$ ) (Fig 2c). The spring-like wire has then been entangled to obtain a rough porous base material (Fig 2d). Those porous samples have finally been placed into a hollow Plexiglas 24-mm inner diameter tube and shaped into final form by applying a compressive force using upper and bottom pistons (Fig 2e).

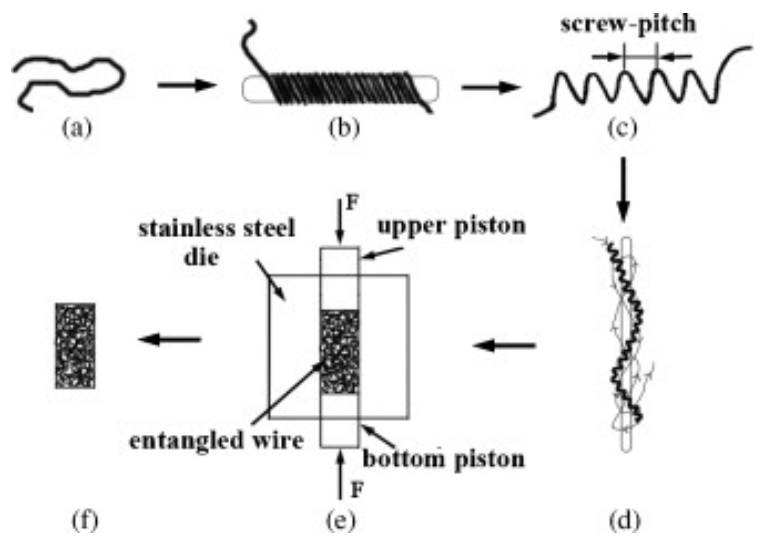

Fig. 2. Schematic diagram of the preparation process (Tan et al. [6]).

\subsection{Mechanical tests}

Quasi-static or dynamic tests have been carried out on the manufactured specimens.

Monotonic quasi-static test were carried out on an electromechanical testing machine (Zwick-Roell $250 \mathrm{kN}$ ) equipped with a $10 \mathrm{kN}$ load sensor. A displacement was applied to the specimen placed between two platens so that the strain rate reached $0.005 \mathrm{~s}^{1}$. Tests were stopped when load was equal to $8 \mathrm{kN}$ (which was reached in the densification region of the stress-strain curve).

High strain rate tests were carried out using compression Split Hopkinson Pressure Viscoelastic 38-mm diameter Bars. Dispersion and attenuation were taken into account using Bacon procedure [11]. Classically, pressure is applied to a striker which hits a viscoelastic input bar, where strains and strain rate are measured from strain gauges. Specimen is positioned between input and output bars, where strains gauges also allow to measure strain levels. Strain rate is then determined at the end of the test.

From all tests, nominal stress, engineering strain and energy were calculated and used as posttreatment parameters. 


\section{Results}

From mechanical tests, Fig. 3 presents nominal stress as a function of engineering strain material response for the two chosen volume fractions and both strain rates. As expected, the mechanical response of the specimen presents typical response of cellular material, i.e., a first linear response, followed by a plastic stress plateau where strain values are increasing while small changes of stress occur, and finally a densification region. Strain rate plays an important role in the mechanical response of the specimen: the stress level of the beginning of the stress plateau strongly depends on both the volume fraction and the strain rate. Considering that a possible application of the material is energy dissipation, absorbed energy per volume unit is plotted as a function of strain on Fig. 4.
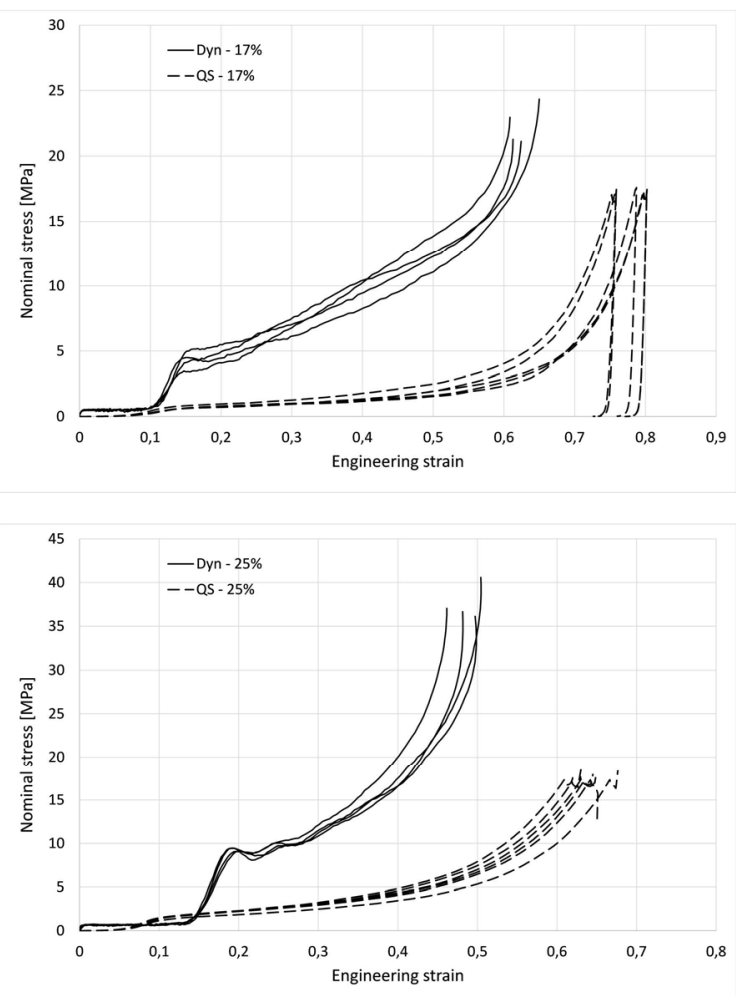

Fig. 3. Stress-Strain curves for the two volume fractions: (top) $\mathrm{Vf}=17 \%$, (bottom) $\mathrm{Vf}=25 \%$.

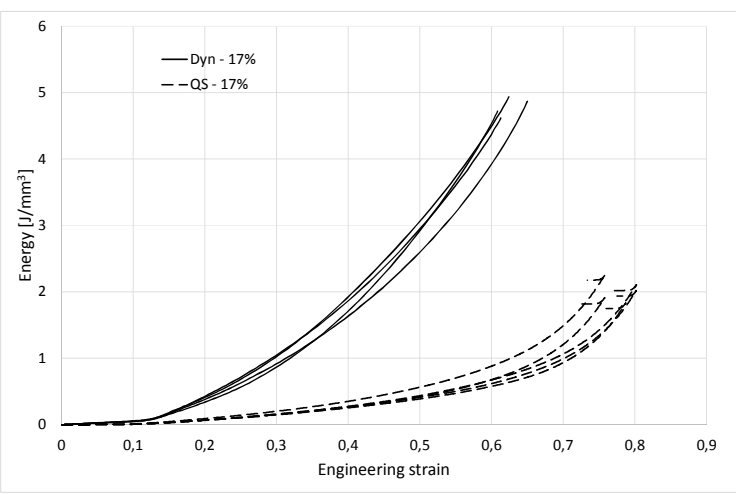

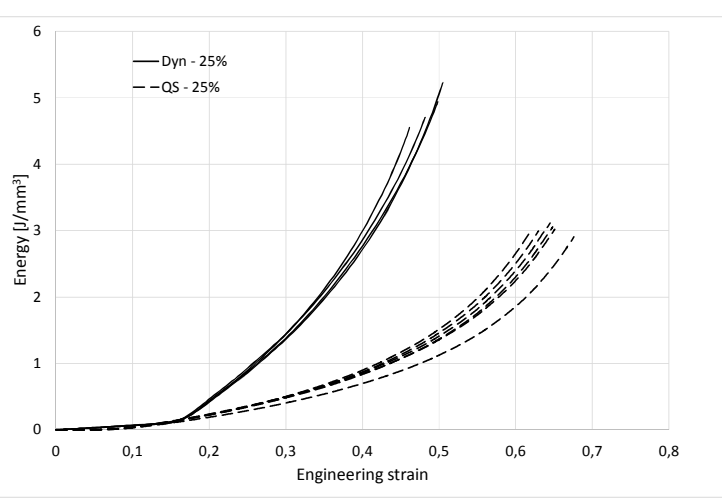

Fig. 4. Absorbed energy versus engineering strain: (top) $\mathrm{Vf}=17 \%$, (bottom) $\mathrm{Vf}=25 \%$.

\section{Discussion}

Fig. 5 makes a comparison between the two densities regarding the absorbed energy at $40 \%$ of strain. As expected, the SWEM is more absorbent with a higher density. The strain rate effect is clearly observed as mentioned in all the previous works of literature and confirms the good suitability of SWEM for shock absorption.

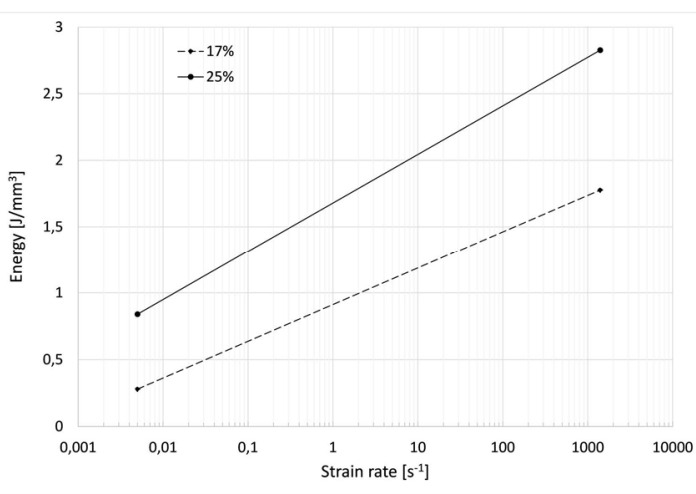

Fig. 5. Absorbed energy at $40 \%$ strain versus strain rate for the two densities.

Regarding the results of this preliminary experimental campaign, several comments can be pointed out:

- The sample slenderness used in this work is valid for dynamic tests. No shear strain was observed during the static or dynamic tests. It is noticed that two different sample heights were used such that further analyses on the size effect in the SWEM must be done;

- The good repeatability for all strain rates and densities validates the manufacturing process that is "handmade". A recent proposal [8] about using weaving techniques could be very useful to decrease time production of a sample (currently equal to one hour for one sample);

- More tests at different strain rates are required to clearly identify the strain rate influence of SWEM. A specific flywheel device [12] could be 
very useful to perform constant strain rate tests and to obtain results at intermediate strain rate.

Finally, the main question being the part of the friction in the shock absorption, it is needed to perform a simulation at the structure level. This simulation needs to take into account all contacts in the material and to handle high strains (up to 60\%) during a dynamic compression loading. Discrete approach was already used to model SWEM [13] by modelling the wire with a coarse "pearl necklace" meshing. Recent work for woven material and dry fabrics using discrete methods [14] could be used to improve representation of the material entanglement.

\section{Conclusions}

The SWEM was investigated under dynamic compression loading with two densities. Although results confirm the good performance of this material for dynamic applications, the present work only presents preliminary results. A more detailed experimental campaign is currently under process using standard Hopkinson bar device but also a specific flywheel compression test, in order to bring more information on the global strain rate influence. Such data will be used to validate a modelling of this material at the wire scale using the Discrete Element Method. Then, this model will be very helpful to analyse the proportion of absorbed energy coming from friction, such that an optimization for dynamic application can be considered like sandwich-core material or even momentum trap device application.

\section{References}

1. D.E. Chegodaev, Design of Metal Rubber Components, Press of National Defense Industry, pp. 2-12 (translated by Li Z.Y., 2000)

2. A.O. Hong-Rui, H.Y. Jiang, H. Yan, et al., Research of a metal rubber isolation system based on complex stiffness, Journal of Harbin Institute of Technology 37, pp. 1615-1629 (in Chinese, 2005)

3. E. Piollet, D. Poquillon, G. Michon, Dynamic hysteresis modelling of entangled cross-linked fibres in shear, Journal of Sound and Vibration 383, pp. 248-264 (2016)

4. D. Rodney, B. Gadot, O.R. Martinez, S. Rolland Du Roscoat, L. Orgéas, Reversible dilatancy in entangled single-wire materials. Nature Materials 15, pp. 72-77 (2015)
5. J.F. Hou, H.B. Bai, D.W. Li, Damping capacity measurement of elastic porous wiremesh material in wide temperature range. Journal of Materials Processing Technology 206, pp. 412-418 (2008)

6. Q. Tan, G. He, Stretching behaviors of entangled materials with spiral wire structure. Materials \& Design 46, pp.61-65 (2013)

7. B. Gadot, O. Riu Martinez, S. Rolland Du Roscoat, D. Bouvard, D. Rodney, L. Orgéas, Entangled single-wire NiTi material: A porous metal with tunable superelastic and shape memory properties, Acta Materialia 96, pp. 311$323(2015)$

8. J. Hu, Q. Du, J. Gao, J. Kang, B. Guo, Compressive mechanical behavior of multiple wire metal rubber, Materials and Design 140, pp. 231-240 (2018)

9. L. Courtois, E. Maire, M. Perez, D. Rodney, O. Bouaziz, Y.Brechet, Mechanical properties of monofilament entangled materials, Optical Measurements, Modeling, and Metrology, 5. ( Springer, New York, NY, 2011)

10. P. Qiao, M. Yang, F. Bobaru, Impact Mechanics and High-Energy Absorbing Materials: Review, Journal of Aerospace Engineering, 21, pp. 235248 (2008)

11. C. Bacon, An experimental method for considering dispersion and attenuation in a viscoelastic Hopkinson bar, Experimental Mechanics, 38, pp. 242-249 (1998)

12. C. Froustey, M. Lambert, J.L. Charles, J.L. Lataillade, Design of an impact loading machine based on a flywheel device: Application to the fatigue resistance of the high rate pre-straining sensitivity of aluminium alloys, Experimental Mechanics, 47, pp. 709-721 (2007)

13. D. Rodney, M. Fivel, R. Dendievel, Discrete modeling of the mechanics of entangled materials, Physical Review Letters, 95 (2005)

14. J. Girardot, F. Dau, A mesoscopic model using the discrete element method for impacts on dry fabrics, Matériaux \& Techniques 104,408 (2016) 